\title{
Clinical features of invasive bronchial-pulmonary aspergillosis in critically ill patients with chronic obstructive respiratory diseases: a prospective study
}

Hangyong $\mathrm{He}^{1+}$, Lin Ding ${ }^{1+}$, Fang $\mathrm{Li}^{2}$, Qingyuan Zhan ${ }^{1 *}$

\begin{abstract}
Introduction: Critically ill patients with chronic obstructive respiratory diseases (CORD) who require intensive care unit (ICU) admission are at particular risk for invasive bronchial-pulmonary aspergillosis (IBPA). The purpose of this study is to investigate clinical features for rapid recognition of IBPA in critically ill patients with CORD.

Methods: We included 55 consecutive CORD patients in a respiratory ICU in a prospective, single-center, cohort study. In this study, IBPA combined two entities: ATB and IPA.

Results: Thirteen of 55 patients were diagnosed with IBPA. Before ICU admission, three variables were independent predictors of IBPA with statistical significance: more than three kinds of antibiotics used before the ICU admission, accumulated doses of corticosteroids (>350 mg) received before the ICU admission, and APACHE II scores $>18$ (OR, 1.208; $P=0.022 ; \mathrm{OR}, 8.661 ; P=0.038$; and $\mathrm{OR}, 19.488 ; P=0.008$, respectively). After ICU admission, more IBPA patients had a high fever $\left(>38.5^{\circ} \mathrm{C}\right)(46.2 \%$ versus $11.9 \% ; P=0.021)$, wheeze without exertion $(84.6 \%$ versus $50.0 \% ; P=0.027)$, dry rales (84.6\% versus $40.4 \% ; P=0.005)$, higher white blood cell counts $\left(21 \times 10^{9} / \mathrm{L}\right.$ versus $\left.9.4 \times 10^{9} / \mathrm{L} ; P=0.012\right)$, lower mean arterial pressures $(77.9 \mathrm{~mm} \mathrm{Hg}$ versus $90.5 \mathrm{~mm} \mathrm{Hg} ; P=0.019)$, and serum creatinine clearances (36.2 ml/ min versus $68.8 \mathrm{ml} / \mathrm{min} ; P<0.001$ ), and liver-function and coagulation abnormalities. Bronchospasm, sputum ropiness, and plaque formation were more common for IBPA patients during bronchoscopy $(66.7 \%$ versus $14.3 \%$; $P=$ $0.082 ; 18 \%$ versus $0 ; P=0.169$; and $73 \%$ versus $13 \% ; P=0.003$, respectively). More IBPA patients had nodules and patchiness on chest radiograph on day 1 of admission, which rapidly progressed to consolidation on day 7 . IBPA mortality was higher than that of non-IBPA patients (69.2\% versus $16.7 \%$; $P=0.001)$.

Conclusions: IBPA may be suspected in critically ill CORD patients with respiratory failure and clinical and bronchoscopic manifestations of severe infection, bronchospasm, and rapid progression of radiologic lesions that are irresponsive to steroids and antibiotics. To avoid misdiagnosis and establish the microbiologic etiology, early bronchoscopy and tight radiologic follow-up should be performed.
\end{abstract}

\section{Introduction}

Aspergillus tracheobronchitis (ATB) and invasive pulmonary aspergillosis (IPA) are two clinical presentations of invasive aspergillosis (IA) [1]. The predisposing factors for ATB and IPA are similar [1,2]. Neutropenic and

\footnotetext{
* Correspondence: cyh_birm@sina.com

+ Contributed equally

'Department of Respiratory and Critical Care Medicine, Beijing Institute of Respiratory Medicine, Beijing Chao-Yang Hospital, Capital Medical University,

8 Gongren Tiyuchang South Road, Beijing, 100020, PR China

Full list of author information is available at the end of the article
}

immunocompromised patients are particularly at risk. Chronic obstructive respiratory disease (CORD) is defined as chronic obstructive diseases of the airways and pulmonary tissues. CORD includes a wide array of serious diseases, and chronic obstructive pulmonary disease (COPD), bronchial asthma, and bronchiectasis are common CORDs [3]. Patients with CORD frequently experience acute exacerbations of their underlying illnesses that require hospitalization or intensive care unit (ICU) admission. Recent reports suggest that the incidence of IA appears to
C Biomed Central 
be increasing in CORD patients requiring ICU admission [4-6]. Moreover, CORD was a major component of critically ill patients with IA in the ICU. Despite invasive ventilation and antifungal treatments, the mortality due to IPA for critically ill COPD patients remains at $67 \%$ to $100 \%$ [2,6-10], and the mortality for ATB is $80 \%$ [2].

These high mortality rates may be the result of unclear clinical features and the delayed diagnoses and treatments for ATB and IPA among CORD patients. Recently, Tasci et al. [2] described the clinical features of ATB and proposed an optimal diagnostic strategy. Bulpa et al. [7] proposed a series of diagnostic criteria for IPA in the COPD population, which provided the criteria for the clinical diagnosis of IPA and ATB. However, these results were based on retrospective studies [2,7].

Several reports have suggested that ATB might progress to or coexist with IPA $[11,12]$. A recent study showed that ATB could occur in moderately or nonimmunocompromised patients with impaired airway structures or defense functions and may be an early stage of IPA [13]. ATB and IPA might be two phases or manifestations of one entity, invasive bronchial-pulmonary aspergillosis (IBPA), which was rarely recognized before. In the present study, we preferred to combine IPA and ATB as one disease, and we used the term IBPA to indicate these two subentities.

The aim of this single-center prospective cohort study was to describe the early clinical signs and to evaluate the available diagnostic procedures for IBPA in critically ill CORD patients in our ICU to assess their importance for rapid recognition and appropriate treatment.

\section{Materials and methods \\ Study population and data collection}

In our study, all of the patients were admitted to an ICU because of respiratory failure from February 2007 to November 2008. These patients were older than 18 years and had been diagnosed with either severe COPD, stage III or IV according to the Global Initiative for Chronic Obstructive Lung Disease (GOLD), moderate or severe persistent bronchial asthma according to Global Initiative for Asthma (GINA), or bronchiectasis with respiratory failure according to their clinical history, symptoms, signs, and laboratory findings.

The following information was stored in a data file: patients' characteristics, including age, sex, medical history, and reasons for ICU admission; use of immunosuppressive drugs (steroids and others) and antibiotics; presence of typical symptoms and signs; and standard ICU laboratory findings on days 1, 4, and 7 after admission, including complete blood count, arterial blood gas analysis (ABGA), serum biochemistry tests, activated partial thromboplastin time (APTT), and microbiologic examination; and disease severity, assessed according to the Acute Physiology and Chronic Health Evaluation II (APACHE II) on their admission to the ICU.

A sandwich enzyme-linked immunosorbent assay (ELISA) for galactomannan (GM) detection (Platelia Aspergillus; Sanofi Diagnostics Pasteur, Marnes-La-Coquette, France) was used according to the manufacturer's instructions. Serum sampling for GM detection was done on days 1, 4, and 7 after ICU admission. An optical density (OD) ratio of 0.5 or greater for GM in serum was considered positive.

Fiberoptic bronchoscopy with bronchoalveolar lavage was performed on days 1, 4, and 7 of inclusion if the patient was intubated and if feasible. The selection of sampling areas was based on the infiltrate location on a chest radiograph. The presence of any tracheal or bronchial lesions was recorded by the endoscopist (QZ). Lavage samples were submitted for direct microscopic examination and bacterial and fungal cultures.

A chest radiograph (CXR) by bedside was done on days 1,4 , and 7 after ICU admission. Pulmonary computed tomography (CT) was also done if feasible, depending on the patients' situations.

Antifungal treatment was started and selected at the discretion of the attending physician (QZ) and was not protocol defined.

The study was approved by the ethics committee, and written, informed consents were obtained from the patients or their next of kin.

\section{Processing of clinical samples}

LRT samples of all patients included in this study were taken once a day for the first 3 days of their ICU stays. LRT samples were collected again once per week if the patient remained in the ICU for more than 7 days. All LRT specimens were cultured on conventional media, including sheep-blood agar and chocolate agar. At the same time, all LRT specimens were cultured on CHROMagar medium and Sabouraud dextrose agar. Cultures were incubated at $25^{\circ} \mathrm{C}$ and $37^{\circ} \mathrm{C}$, respectively, for 7 to 14 days. When spore growth was suboptimal on the routine media, LRT samples were further cultured on potato dextrose agar for a better conidial production. Aspergillus isolates were identified by using standard morphologic procedures, including colonial morphology, growth velocity, color, morphology of hyphae, and characteristics of hyphae and spores under microscopy.

\section{Case definitions of IBPA}

According to the definitions of invasive fungal disease of the European Organization for Research and Treatment of Cancer/Mycoses Study Group (EORTC/MSG), ATB is diagnosed when tracheobronchial ulceration, nodule, pseudomembrane, plaque, or eschar is seen on bronchoscopic analysis, which is confirmed by biopsy or positive culture for Aspergillus or both [1]. 
IPA was classified as "proven," "probable," or "possible," based on case definitions of EORTC/MSG [1]. Proven IPA referred to histopathologic evidence of tissue invasion by septated, acutely branching filamentous fungi together with a positive culture. Probable IPA referred to the presence of a positive culture or cytology for Aspergillus species from any lower respiratory tract (LRT) sample together with one major criterion (halo sign, air-crescent sign, or cavity within an area of consolidation on CT scan) or two of three minor clinical criteria (symptoms of LRT infection, pleural rub, or new infiltrate without an alternative diagnosis). Possible infection referred to patients who fulfilled probable infection criteria but did not have a positive Aspergillus culture or microscopy from LRT, or serology. Patients with positive cultures for Aspergillus from nonsterile sites, but without any other evidence of fungal infection, were considered to be colonized. Diagnosis was not based on a serum GM test.

The diagnosis of IBPA referred to a patient diagnosed with ATB or IPA or both. Patients diagnosed with ATB or IPA or both were included in the IBPA group. Among critically ill patients with COPD, bronchial asthma, and bronchiectasis, those diagnosed as nonIBPA were included in the non-IBPA group. Patients with Aspergillus colonization were considered noninfected cases and were included in the non-IBPA group.

\section{Statistical analysis}

Patients with CORD admitted to the ICU were divided into IBPA and non-IBPA groups. The clinical signs and results of diagnostic tests were compared between the two groups. Normally distributed continuous variables were expressed as mean $\pm \mathrm{SD}$ and compared with a $t$ test. Non-normally distributed continuous variables were expressed as median and quartiles and compared with the Wilcoxon rank-sum test. Categoric variables were compared with a $\chi^{2}$ test. Multivariate logisticregression analysis was used to identify independent risk factors for IBPA patients. The $P$ values $<0.05$ were considered significant. All analyses were carried out with the use of SPSS software for Windows (release 11.5).

\section{Results}

\section{Patient characteristics}

From February 2007 to November 2008, in total, 343 patients were admitted to our ICU. Fifty-five of these patients who met the inclusion criteria were enrolled: 47 (86\%) had COPD, four (7\%) had asthma, and four (7\%) had bronchiectasis. The characteristics of the total study group are shown in Table 1.

Thirteen (24\%) patients were diagnosed with IBPA, and the remaining patients (42) did not have IBPA. In IBPA group, 11 patients had COPD, one patient had asthma, and one patient had bronchiectasis. According to the diagnostic criteria for IBPA, the 13 IBPA patients were classified as proven $(n=4)$, probable $(n=8)$, and possible IBPA $(n=1)$ (Table 2). One case was diagnosed as colonized. Aspergillus spp. was the only mold pathogen, and no other non-Aspergillus invasive mold infection was found in the patients studied. IBPA cases were diagnosed at a median of 2 days (IQR, 1 to 7 days) after the patients' admission to the ICU. The reasons for respiratory failure in IBPA patients were infection (12 cases) and heart dysfunction (one case), which caused exacerbations of their underlying respiratory diseases. Eleven of the 13 IBPA patients had a positive culture or microscopic examination of Aspergillus spp. for their LRT samples collected at the first day of their ICU admission, and two patients had positive microbiologic results for LRT samples collected at day 6 and 8 after the ICU admission. As a result, these 11 cases were determined as having developed the infection before the ICU admission. All IBPA patients and 25 non-IBPA patients received invasive mechanical ventilation $(100 \%$ versus $59.5 \% ; P=0.016)$. The duration of invasive mechanical ventilation for IBPA was significantly longer than that for non-IBPA patients ( 8 days versus 3 days; $P=0.006)$. The mortality for IBPA was higher than that of control group $(69.2 \%$ versus $16.7 \%$; $P=0.001)$. The causes of death for these nine IBPA patients were multiple organ failure for four cases, acute renal failure for three cases, and septic shock for two cases.

Four patients had tracheobronchial mucus biopsies, and two of them had lung biopsies; no autopsy was obtained for this study.

In the IBPA group, 13 patients had a length of ICU stay of more than 1 day, 12 patients for more than 4 days, and 9 patients for more than 7 days, respectively. In the nonIBPA patients, 42 cases, 36 cases, and 23 cases stayed in the ICU for more than 1,4 , and 7 days, respectively.

\section{Steroids and antibiotics Steroids}

The dosages of systemic steroids received by all patients were converted to prednisone or equivalent doses by steroid potency (for example, $20 \mathrm{mg}$ of hydrocortisone $=5 \mathrm{mg}$ of prednisone). The numbers of patients who received steroids treatment before ICU admission in the IBPA and non-IBPA groups were similar (69\% versus 62\%). Compared with non-IBPA patients, before their admissions to the ICU, IBPA patients received a significantly higher mean dosage of systemic steroids (371 $\mathrm{mg}$ versus $180 \mathrm{mg}$ of prednisone or equivalent; $P=0.006$ ). IBPA patients received steroids for a longer period than did non-IBPA patients (median, 6 days versus 1 day). The median daily dosages of systemic steroids received by IBPA and non-IBPA patients were similar (Table 1). 


\begin{tabular}{|c|c|c|c|}
\hline & IBPA & Control & $P$ value \\
\hline Number of patients & 13 & 42 & -— \\
\hline \multicolumn{4}{|l|}{ Demographic characteristics } \\
\hline Age, mean, years (SD) & $74.3(13.5)$ & $73.2(7.46)$ & 0.150 \\
\hline \multicolumn{4}{|l|}{ Sex, number (\%) } \\
\hline Male & $7(53.8)$ & $25(59.5)$ & 0.228 \\
\hline Female & $6(46.2)$ & $17(40.5)$ & \\
\hline Length of hospitalization before ICU admission, days (IQR) & $15(8.5-29.5)$ & $3(2-6.25)$ & $0.001^{a}$ \\
\hline Length of ICU stay, days (IQR) & $10(6-20)$ & $7(5-14)$ & 0.253 \\
\hline Transferred from other hospital/ICU, number (\%) & $5(38.5)$ & $5(11.9)$ & 0.079 \\
\hline From other ICU, number (\%) & $1(7.7)$ & $3(7.1)$ & 0.672 \\
\hline From other hospital, number (\%) & $4(30.8)$ & $2(4.8)$ & $0.034^{\mathrm{a}}$ \\
\hline \multicolumn{4}{|l|}{ Medical history, number (\%) } \\
\hline Three or more hospitalizations & $9(69.2)$ & $38(90.5)$ & 0.147 \\
\hline Chronic renal dysfunction & $3(23.1)$ & $4(9.5)$ & 0.421 \\
\hline Diabetes mellitus & $0(0)$ & $6(14.3)$ & 0.350 \\
\hline Nonhematologic malignancy & $0(0)$ & $3(7.1)$ & 0.438 \\
\hline \multicolumn{4}{|l|}{ Corticosteroids use } \\
\hline Number of patients with steroids use (\%) & $9(69.2)$ & $26(61.9)$ & 0.881 \\
\hline Prolonged steroids for $\geq 3$ weeks before ICU admission, number (\%) & $1(7.7)$ & $5(11.9)$ & 1.000 \\
\hline 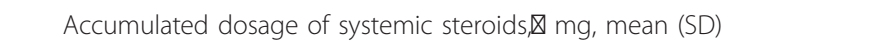 & $371(199)$ & $180(150)$ & $0.006^{\mathrm{a}}$ \\
\hline Accumulated dosage of systemic steroids, $₫$ mg, median (IQR) & $400(190-535)$ & $105(75-241)$ & $0.021^{\mathrm{a}}$ \\
\hline Duration of steroids before ICU admission, day, median (IQR) & $6(0-7)$ & $1(0-3)$ & $0.041^{\mathrm{a}}$ \\
\hline Daily dosage of systemic steroids $\bigotimes$, mg, median (IQR) & $50(0-75)$ & $50(0-63)$ & 0.377 \\
\hline Inhaled steroids, number (\%) & $1(7.7)$ & $2(4.8)$ & 1.000 \\
\hline \multicolumn{4}{|l|}{ Antibiotics } \\
\hline Number of patients with antibiotics (\%) & $13(100)$ & $36(85.7)$ & 0.350 \\
\hline Number of kinds of antibiotics, median (IQR) & $3(1-5)$ & $2(1-2)$ & $0.037^{\mathrm{a}}$ \\
\hline Length of antibiotics use, days, median (IQR) & $10(4.5-22.0)$ & $3(2-10.3)$ & $0.015^{\mathrm{a}}$ \\
\hline APACHE II scores, mean (SD) & $18.6(7.1)$ & $12.6(4.5)$ & $0.010^{\mathrm{a}}$ \\
\hline \multicolumn{4}{|l|}{ Mechanical ventilation } \\
\hline Total number of patients with invasive ventilation during RICU stays (\%) & $13(100)$ & $25(59.5)$ & $0.016^{\mathrm{a}}$ \\
\hline Duration of invasive mechanical ventilation, days, median (IQR) & $8(5-15)$ & $3(0-10)$ & $0.006^{\mathrm{a}}$ \\
\hline \multicolumn{4}{|l|}{ Outcome, number (\%) } \\
\hline Survival & $4(30.8)$ & $35(83.3)$ & $0.001^{\mathrm{a}}$ \\
\hline Dead & $9(69.2)$ & $7(16.7)$ & \\
\hline
\end{tabular}

SD, standard deviation; IQR, interquartile range; APACHE II, Acute Physiology and Chronic Health Evaluation II. खThe steroid doses were converted to prednisone dose (for example, $20 \mathrm{mg}$ of hydrocortisone = approximately $5 \mathrm{mg}$ of prednisone). ${ }^{\mathrm{a}} \mathrm{P}<0.05$.

\section{Antibiotics}

Most patients in the two groups received antibiotics treatment before their ICU admissions. The IBPA patients were given significantly more kinds of antibiotics for a longer treatment period than were the non-IBPA patients (Table 1).

\section{Symptoms and Signs \\ Symptoms}

More IBPA patients had high fevers did non-IBPA patients $\left(\mathrm{T}>38^{\circ} \mathrm{C}\right.$; $46 \%$ versus $12 \%$; $\left.P=0.021\right)$. Compared with non-IBPA patients, wheeze without exertion was a more common symptom for IBPA patients $(85 \%$ versus $50 \%)$. Hemoptysis and chest pain were rare in both groups (Table 3).

\section{Signs}

On admission to the ICU, heart rates and respiratory rates were similar for IBPA and non-IBPA patients. Mean arterial blood pressures were significantly lower for IBPA patients than for non-IBPA patients $(78 \mathrm{~mm}$ $\mathrm{Hg}$ versus $91 \mathrm{~mm} \mathrm{Hg} P=0.019)$. Dry rales were heard more frequently in the lungs of IBPA patients (85\% versus $40 \% ; P=0.005$ ) (Table 3 ).

\section{Multivariate analysis}

Variables with a $P$ value $<0.1$ in the univariate analysis are shown in Tables 1 and 3 . Of these, three were included in the multivariate model: more than three kinds of antibiotics used before the ICU admission, 


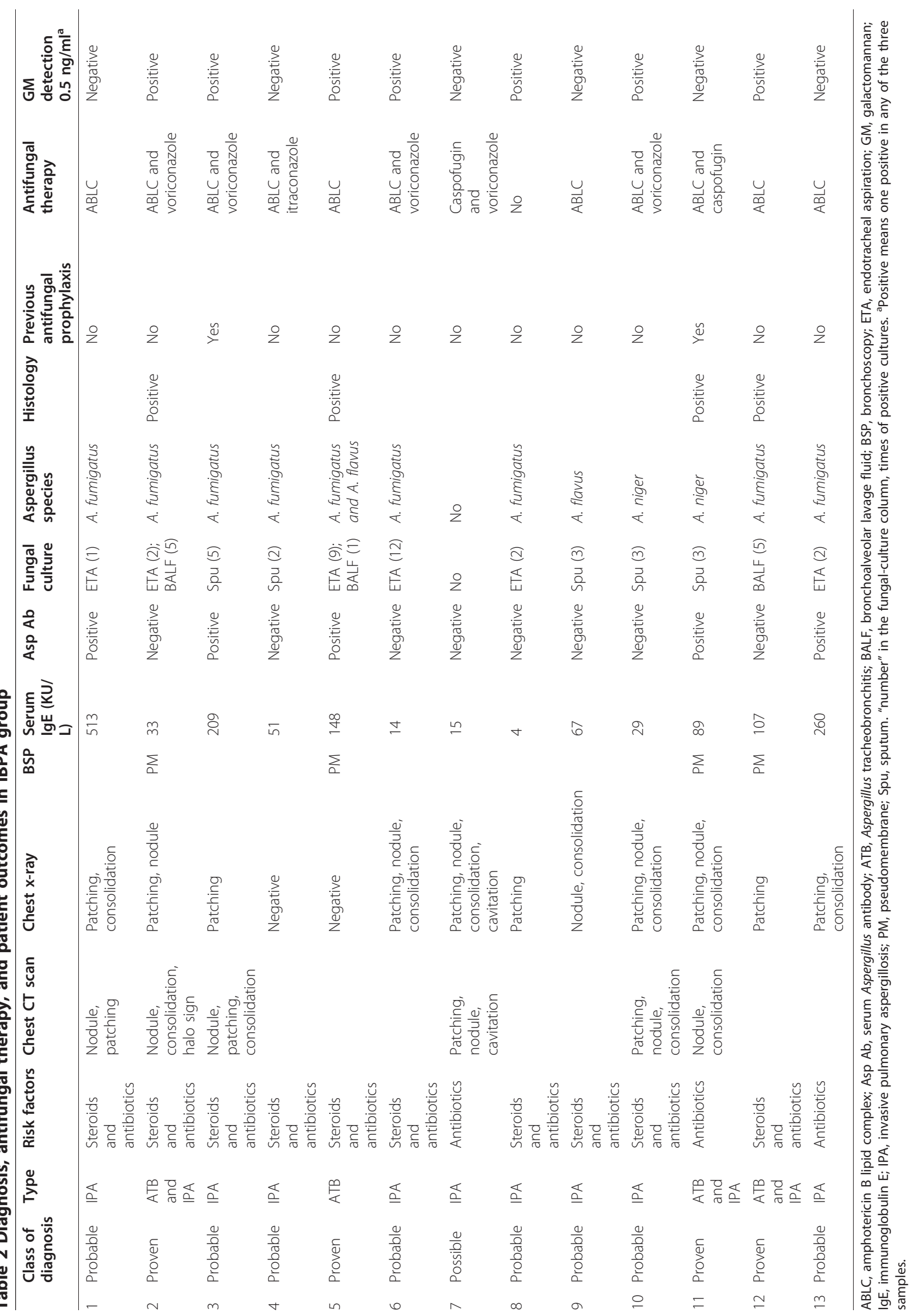


Table 3 Clinical characteristics (symptoms and signs)

\begin{tabular}{|c|c|c|c|}
\hline & IBPA & Control & $P$ value \\
\hline Number of patients & 13 & 42 & -— \\
\hline \multicolumn{4}{|l|}{ Symptoms, number (\%) } \\
\hline Fever & $8(61.5)$ & $10(23.8)$ & $0.028^{a}$ \\
\hline Body temperature $>38.5^{\circ} \mathrm{C}$ & $6(46.2)$ & $5(11.9)$ & $0.021^{a}$ \\
\hline Cough & $10(76.9)$ & $36(85.7)$ & 0.749 \\
\hline Wheeze & $11(84.6)$ & $36(85.7)$ & 1.000 \\
\hline Wheeze with exertion & $0(0)$ & $15(35.7)$ & $0.030^{\mathrm{a}}$ \\
\hline Wheeze without exertion & $11(84.6)$ & $21(50)$ & $0.027^{\mathrm{a}}$ \\
\hline Sputum production & $10(76.9)$ & $37(88.1)$ & 0.583 \\
\hline Phlegm & $3(23.1)$ & $5(11.9)$ & 0.583 \\
\hline Hemoptysis & $2(15.4)$ & $1(2.4)$ & 0.136 \\
\hline Chest pain & $2(15.4)$ & $2(4.8)$ & 0.234 \\
\hline \multicolumn{4}{|l|}{ Signs at ICU admission } \\
\hline Body temperature, ${ }^{\circ} \mathrm{C}$, mean (SD) & $36.6(0.5)$ & $36.7(0.6)$ & 0.876 \\
\hline Heart rate, beats per minute, mean (SD) & $106.5(23.9)$ & $95.2(21.2)$ & 0.108 \\
\hline Respiratory rate, breaths per minute, mean (SD) & $28.3(8.5)$ & $24.3(11.4)$ & 0.243 \\
\hline Mean arterial pressure, mean (SD) & $77.9(14.2)$ & $90.5(17.0)$ & $0.019^{a}$ \\
\hline \multicolumn{4}{|l|}{ Rales, number (\%) } \\
\hline Dry rales & $11(84.6)$ & $17(40.4)$ & $0.005^{\mathrm{a}}$ \\
\hline Moist rales & $9(69.2)$ & $30(71.4)$ & 1.000 \\
\hline
\end{tabular}

SD, standard deviation; IQR, interquartile range. ${ }^{\mathrm{a}} P<0.05$.

accumulated doses of corticosteroids ( $>350 \mathrm{mg}$ ) received before the ICU admission, and APACHE II scores $>18$. The multivariate analysis selected the three variables with independent statistical significance (Table 4).

\section{Laboratory tests}

White blood cell (WBC) counts were significantly higher for IBPA patients on days 1, 4, and 7 of ICU admission. The $\mathrm{pH}$ and base excess (BE) were significantly lower for IBPA patients on the first day, but were not different on days 4 and 7 . Serum creatinine clearances were significantly decreased for IBPA compared with non-IBPA patients on days 1, 4, and 7 of ICU admission. During their ICU stays, IBPA patients had significantly higher serum aspartate aminotransferase levels, alanine aminotransferase levels, and activated partial thromboplastin times (see Table 5, Figure $\mathrm{S} 1$ in Additional file 1, and Figure S2 in Additional file 2).

\section{Fiberoptic bronchoscopy}

On days 1, 4, and 7 after ICU admission, 11, 10, and four IBPA patients and 15, six, and three non-IBPA patients had bronchoscope examinations, respectively. For IBPA patients, mucous hyperemia and edema were observed, ropy sputum was difficult to suck out, and four cases showed pseudomembrane formation under bronchoscopic analysis. Bronchospasm, plug formation and sputum ropiness were more common for IBPA on the first day after ICU admission (66.7\% versus $14.3 \%$; $P=0.082 ; 18 \%$ versus $0 ; P=0.169$; and $73 \%$ versus $13 \%$; $P=0.003$, respectively). Four patients in the IBPA group had biopsies of the tracheobronchial tree during bronchoscopy, which showed Aspergillus invasion into the tracheobronchial wall.

\section{Radiologic examination}

On days 1, 4, and 7 after ICU admission, 13, 12, and nine IBPA patients and 42, 32, and 22 non-IBPA

Table 4 Variables selected for prediction of invasive bronchopulmonary aspergillosis by multivariate logistic regression analysis in patients with chronic obstructive respiratory disease

\begin{tabular}{|c|c|c|c|c|c|}
\hline & \multirow[t]{2}{*}{ Wald } & \multirow[t]{2}{*}{$P$ Value } & \multirow[t]{2}{*}{ Odds ratio } & \multicolumn{2}{|c|}{ 95\% Confidence interva } \\
\hline & & & & Inferior & Superior \\
\hline Accumulated dosage of systemic steroids (>350 mg) received before the ICU admission & 4.326 & 0.038 & 8.661 & 1.133 & 66.239 \\
\hline More than three kinds of antibiotics before the ICU admission & 5.211 & 0.022 & 1.208 & 1.027 & 1.422 \\
\hline APACHE $\|$ scores $>18$ & 6.974 & 0.008 & 19.488 & 2.150 & 176.613 \\
\hline Constant & 11.912 & 0.001 & & & \\
\hline
\end{tabular}

APACHE, Acute Physiology and Chronic Health Evaluation; ICU, intensive care unit. 
Table 5 Laboratory findings

\begin{tabular}{|c|c|c|c|c|}
\hline & Day of ICU admission & IBPA & Control & $P$ value \\
\hline \multicolumn{5}{|l|}{ Complete blood count } \\
\hline \multirow[t]{3}{*}{ White blood cell count $\left(\times 10^{9} / L\right)$, mean (SD) } & Day 1 & $21.0(14.0)$ & $9.4(3.7)$ & $0.012^{a}$ \\
\hline & Day 4 & $17.5(5.6)$ & 10.6(13.9) & 0.101 \\
\hline & Day 7 & 19.5(6.3) & $10.0(5.4)$ & $0.000^{\mathrm{a}}$ \\
\hline \multirow[t]{3}{*}{ Neutrophilic granulocyte (\%), mean (SD) } & Day 1 & $90.2(6.3)$ & $84.3(10.0)$ & 0.053 \\
\hline & Day 4 & $89.4(7.7)$ & $79.3(10.7)$ & $0.004^{\mathrm{a}}$ \\
\hline & Day 7 & $87.8(6.9)$ & $80.3(8.6)$ & $0.027^{a}$ \\
\hline \multicolumn{5}{|l|}{ Arterial blood gas analysis } \\
\hline \multirow[t]{3}{*}{$\mathrm{pH}$, mean $(\mathrm{SD})$} & Day 1 & 7.25(0.14) & 7.36(0.11) & $0.005^{\mathrm{a}}$ \\
\hline & Day 4 & $7.42(0.08)$ & $7.42(0.05)$ & 0.768 \\
\hline & Day 7 & 7.42(0.10) & $7.42(0.05)$ & 0.995 \\
\hline \multirow[t]{3}{*}{$\mathrm{PaCO}_{2}(\mathrm{~mm} \mathrm{Hg})$, mean (SD) } & Day 1 & $65.3(36.1)$ & $68.8(33.1)$ & 0.745 \\
\hline & Day 4 & $53.3(22.7)$ & $53.5(11.4)$ & 0.979 \\
\hline & Day 7 & $47.9(22.4)$ & $49.2(12.9)$ & 0.845 \\
\hline \multirow[t]{3}{*}{ Ratio of the $\mathrm{PaO}_{2}$ to $\mathrm{FiO}_{2}$, mean (SD) } & Day 1 & 166.0(86.8) & $219.1(128.8)$ & 0.171 \\
\hline & Day 4 & 197.5(80.0) & $225.7(88.2)$ & 0.332 \\
\hline & Day 7 & 199.9(72.3) & 236.8(83.8) & 0.255 \\
\hline \multicolumn{5}{|l|}{ Renal function } \\
\hline \multirow[t]{3}{*}{ Clearance of creatinine $(\mathrm{ml} / \mathrm{min})$, mean (SD) } & Day 1 & $36.2(20.4)$ & $68.8(27.5)$ & $0.000^{\mathrm{a}}$ \\
\hline & Day 4 & $36.6(24.0)$ & $82.5(51.5)$ & $0.005^{\mathrm{a}}$ \\
\hline & Day 7 & $33.3(32.3)$ & $77.6(50.4)$ & $0.021^{\mathrm{a}}$ \\
\hline \multicolumn{5}{|l|}{ Liver function } \\
\hline $\mathrm{ALT}(\mathrm{U} / \mathrm{L})$, median (IQR) & & $52.5(36.5-95)$ & $28(20-43)$ & $0.003^{\mathrm{a}}$ \\
\hline AST (U/L), median (IQR) & & $67(49-118.5)$ & $26.5(21.8-49.8)$ & $0.000^{\mathrm{a}}$ \\
\hline \multicolumn{5}{|l|}{ Coagulation } \\
\hline $\operatorname{APTT}(\mathrm{s})$, median (IQR) & & $34.8(28.4-49.3)$ & $28.5(26.2-37.7)$ & $0.046^{\mathrm{a}}$ \\
\hline
\end{tabular}

ALT, alanine aminotransferase; APT, activated partial thromboplastin time; AST, aspartate aminotransferase; $\mathrm{FiO}_{2}$, the fraction of inspired oxygen; SD, standard deviation; $\mathrm{IQR}$, interquartile range; $\mathrm{PaCO}_{2}$, partial pressure of arterial carbon dioxide; $\mathrm{PaO}_{2}$, partial pressure of arterial oxygen. ${ }^{\mathrm{a}} \mathrm{P}<0.05$.

patients had radiologic examinations, respectively. In each group, six patients had chest CTs on the first day of ICU admission. Among the six cases with IBPA, one had a halo sign, and one had a cavity on the CT scans. The $\mathrm{CT}$ scans of the other four IBPA cases and the six nonIBPA patients showed nonspecific patching, nodules, and consolidations. The numbers of IBPA patients with nodules and consolidations on CXR increased rapidly from day 1 to day 7 of ICU admission (nodules: from three patients to six patients; consolidations: from one patient to five patients). Compared with non-IBPA patients, patchiness and nodules were more common on CXR on day 1 of admission for IBPA patients (77\% versus $43 \%, P=0.032$; and $23 \%$ versus $2.3 \%, P=0.012$, respectively). At day 4, no significant differences were found between the two groups, and at day 7 , nodules and consolidations were significantly more common for the IBPA patients (60\% versus $9 \%, P=0.002$; and $50 \%$ versus $14 \%$, $P=0.028$ ) (see Figure S3 in Additional file 3).

\section{Serum GM test}

The sensitivities, specificities, positive and negative predictive values, and total consistent rates for positive GM results of a first test and of a second test, at least one positive GM result from two consecutive tests, and both positive GM results of two consecutive tests are shown in Table 6. The total consistent rates did not show significant differences between different diagnostic strategies.

\section{Diagnostic algorithm}

Based on the risk factors, symptoms and signs, and diagnostic procedures evaluated in our study, a diagnostic algorithm is shown in Figure 1.

\section{Discussion}

The main strength of our study is its prospective design that included non-IBPA CORD patients as a control group. This enabled us to discriminate between IBPA in the CORD population and acute exacerbations caused by CORD itself. Our study revealed that before ICU admission, three variables were independent predictors of IBPA: more than three kinds of antibiotics used before the ICU admission, accumulated doses of corticosteroids $(>350 \mathrm{mg}$ ) received before the ICU admission, and APACHE II scores $>18$. In critically ill CORD 
Table 6 Results of first and two consecutive detections of galactomannan in serum of critically ill CORD patients

\begin{tabular}{lllll}
\hline & \multicolumn{2}{c}{ Single GM detection $(\mathbf{9 5 \%} \mathrm{Cl})$} & \multicolumn{2}{c}{ Two consecutive GM detections $(\mathbf{9 5 \%} \mathrm{Cl})(\boldsymbol{n}=\mathbf{4 8})$} \\
\cline { 2 - 5 } & $\begin{array}{l}\text { Positive for a first test } \\
(\boldsymbol{n}=\mathbf{5 5})\end{array}$ & $\begin{array}{l}\text { Positive for a second test } \\
(\boldsymbol{n}=\mathbf{4 8})\end{array}$ & $\begin{array}{l}\text { At least one positive of the two } \\
\text { consecutive tests }\end{array}$ & $\begin{array}{l}\text { Both positive for the two } \\
\text { consecutive tests }\end{array}$ \\
\hline $\begin{array}{l}\text { Sensitivity } \\
(\%)\end{array}$ & $46.2(33.3-59.1)$ & $50.0(35.9-54.1)$ & $53.8(39.7-67.9)$ & $41.7(27.8-55.6)$ \\
$\begin{array}{l}\text { Specificity } \\
(\%)\end{array}$ & $83.3(73.4-93.2)$ & $93.5(86.5-100)$ & $81.0(69.9-92.1)$ & $93.5(86.5-100)$ \\
PPV (\%) & $46.2(33.3-59.1)$ & $75.0(62.7-87.3)$ & $46.7(32.6-60.8)$ & $71.4(58.7-84.1)$ \\
NPV (\%) & $83.3(73.4-93.2)$ & $82.9(72.2-93.6)$ & $85.0(74.9-95.1)$ & $80.6(69.7-91.5)$ \\
TCR (\%) & $74.5(63.0-86.0)$ & $81.4(70.4-91.4)$ & $74.5(62.2-86.8)$ & $79.1(67.9-90.3)$ \\
\hline
\end{tabular}

CORD, chronic obstructive respiratory disease; NPV, negative predictive value; PPV, positive predictive value; TCR, total consistent rate.

patients, IBPA may present as respiratory failure and clinical and bronchoscopic manifestations of severe infection, bronchospasm, and rapid progression of radiologic lesions that are unresponsive to steroids and antibiotics.

In CORD patients, because the structures and defense functions of the airways and lung parenchyma are damaged by their underlying respiratory diseases,
Aspergillus may colonize in these sites. During the early period of invasive aspergillosis, infection may be limited to the tracheobronchial tree, presenting as ATB. This may account for the obvious bronchospasm without radiologic appearance in some cases during the early phase of infection. With corticosteroids and broad-spectrum antibiotics therapy, the infection could spread to the distal airways and lung parenchyma, presenting as

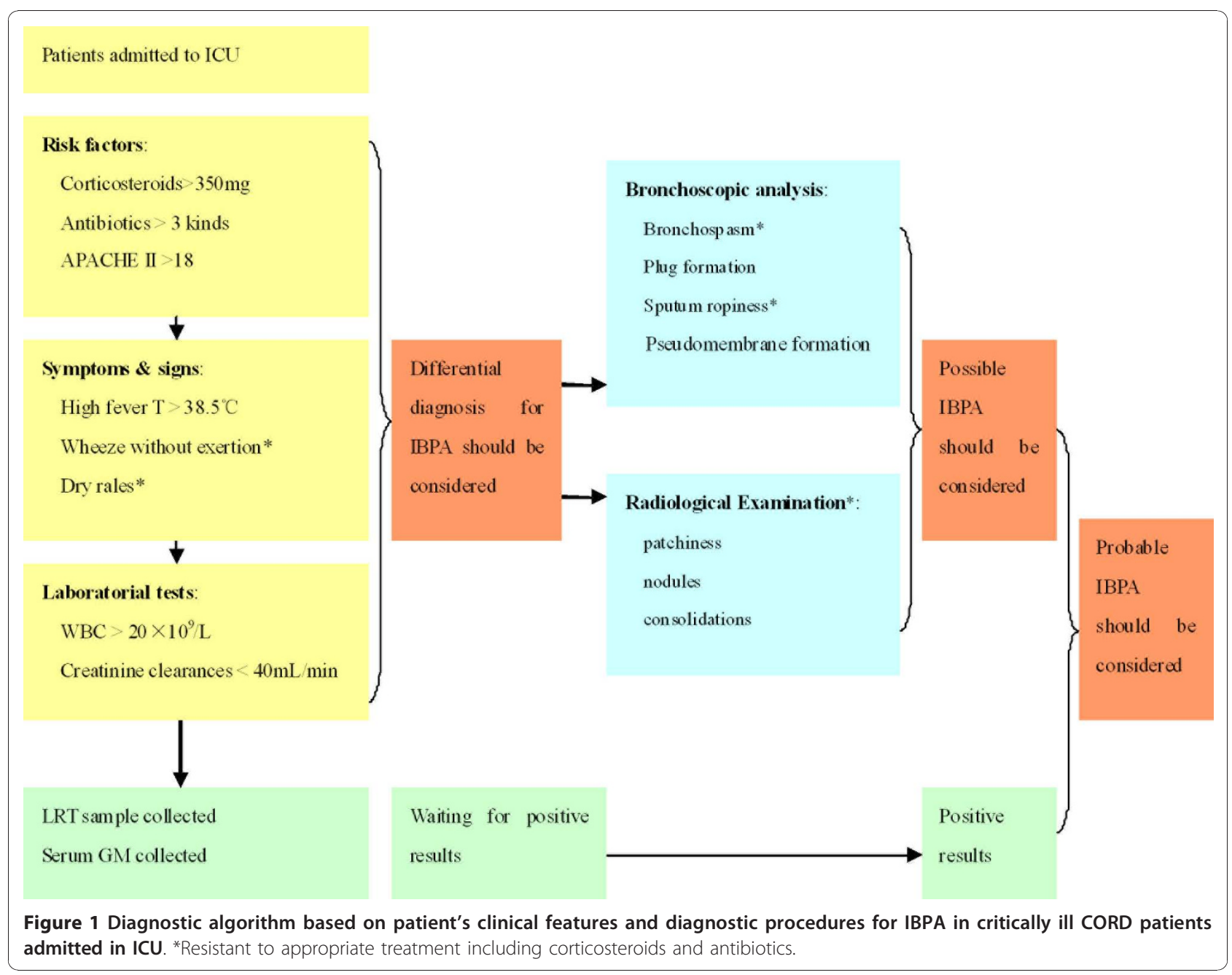


IPA. Several reports have shown that lung parenchyma was usually involved together with ATB, and invasive ATB may indicate an advanced pulmonary lesion caused by Aspergillus [11-15]. In our study, two patients who had a tracheobronchial mucus and lung biopsy (cases 2 and 12) had specific radiologic findings on their CT scans, as well as positive GM tests. In addition, in case 2 , the lesions of the airways and lung parenchyma responded to antifungal treatment, which suggested a concomitant pulmonary lesion secondary to Aspergillus (see Figure 2). Therefore, ATB may be an early stage of IPA, and may exist either before or with IPA.

For CORD patients, corticosteroids treatment is considered to be an important risk factor for IBPA
$[4,7,10,16]$. The conicidal activity of human tissue macrophages is responsible for the monocyte-mediated damage to fungal hyphae [17], and this immune function could be impaired by corticosteroids [18]. Moreover, corticosteroids promote the in vitro growth of Aspergillus fumigatus [19]. Previous studies reported that COPD patients who were given an average daily dose of systemic corticosteroids greater than 73 to 80 $\mathrm{mg}$ of prednisone (or equivalent), and with an average therapy duration of 29.7 days to 2 months were prone to developing invasive aspergillosis $[6,8,20]$. However, another retrospective study showed that an accumulated dosage of steroids equivalent to $>700 \mathrm{mg}$ of prednisone received during the 3 months before hospital admission
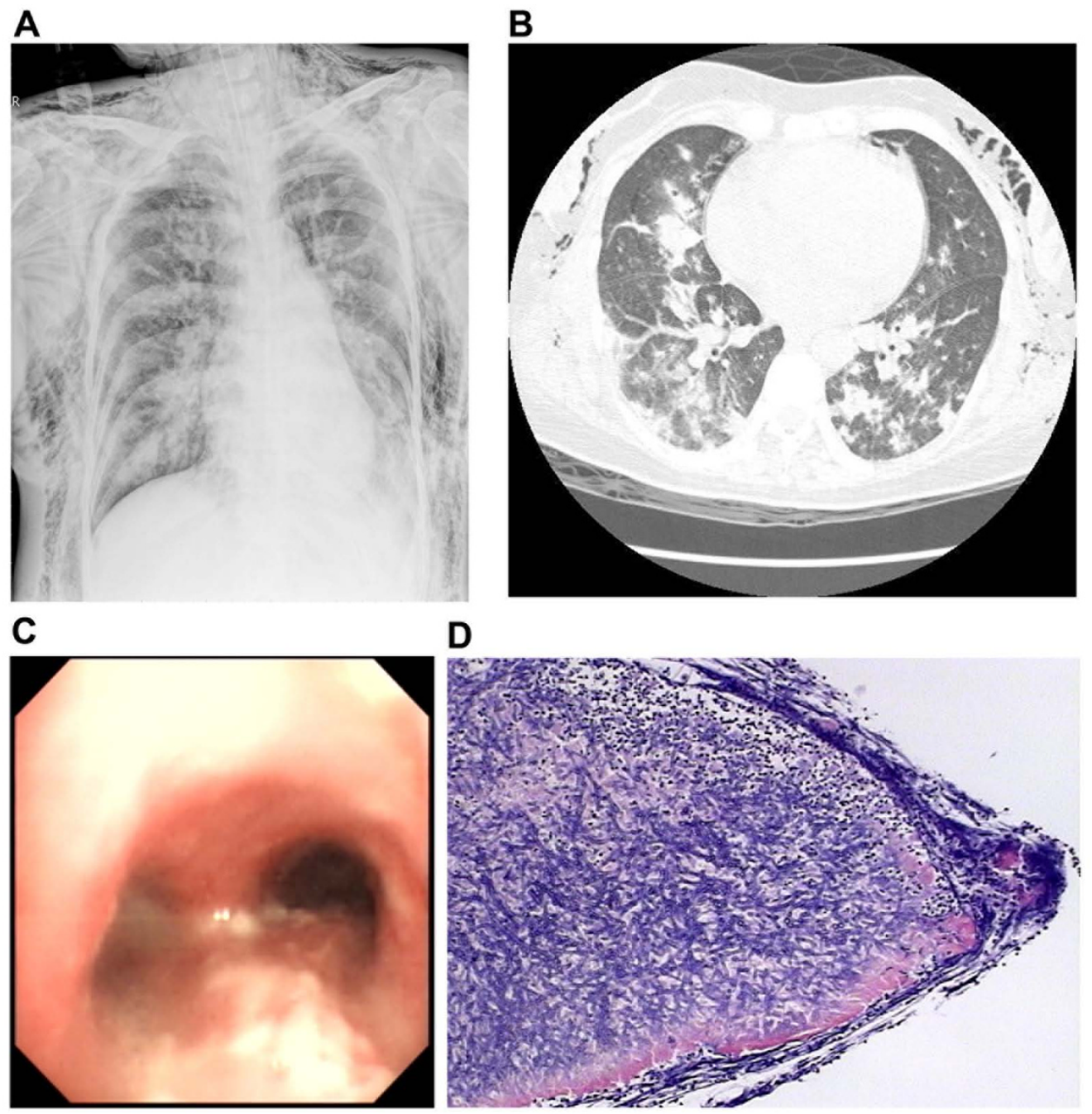

Figure 2 Radiologic, bronchoscopic, and histologic information of Patient IBPA Case 2. (a) Chest x-ray shows patchings and multiple nodules in bilateral lungs, with severe barotrauma. (b) Chest HRCT shows patchings and multiple nodules with halo sign in bilateral lungs. (c) Bronchoscopy shows inflammation and white plaques formation of the airway. (d) Histologic examination of biopsy specimen from lung tissue shows many Aspergillus hyphae invading the lung parenchyma. 
may be a risk factor for IPA in COPD patients [21]. In our study, the patients in the IBPA and non-IBPA groups received the same daily dosages of steroids. However, because the IBPA patients received them for a longer period (median, 6 days versus 1 day), the accumulated steroid dosages in this group may have been higher than those in the non-IBPA group. According to the multivariate analysis in our study, an accumulated dosage of $350 \mathrm{mg}$ prednisone may be associated with IBPA in critically ill CORD patients. For the two IBPA patients who had positive microbiologic results for LRT samples collected at days 6 and 8 after the ICU admission, no corticosteroids were used after their ICU admission, which means that for the 13 IBPA cases, the "accumulated dosage before the admission to the ICU" was the same as "accumulated dose of corticosteroids before the first isolation of Aspergillus" proposed in a previous study [21].

Antibiotic therapy before admission to an ICU could also be an important risk factor. This was observed, but not confirmed, in two retrospective studies $[8,22]$. Muquim et al. [20] reported that IPA that occurred with COPD was associated with the use of multiple broad-spectrum antibiotics before patients' hospitalizations, and the risk for IPA increased with the number of antibiotics used. The number of antibiotics used may suggest a pneumonia that did not respond to several antibiotic treatments. In our study, a median of three or more antibiotics for 10 days may have been a risk factor for IBPA for the critically ill CORD patients before their ICU admissions.

CORD patients are not immunocompromised, although some of them may be mildly immunosuppressed. Therefore, when their airways or lung parenchyma are invaded by Aspergillus, their immune systems should react to this pathogen, and they may show a severe systemic inflammatory reaction [7] and obvious bronchospasm [23]. High fever $\left(\mathrm{T}>38.5^{\circ} \mathrm{C}\right)$ and elevated WBC $\left(>20 \times 10^{9} / \mathrm{L}\right)$ are systemic manifestations of inflammatory reactions. The trend of low arterial pressure, acidosis, acute renal dysfunction (creatinine clearance $<36 \mathrm{ml} / \mathrm{min}$ ), and abnormalities of liver function and coagulation in critically ill CORD patients may suggest the presence of a more severe inflammatory status, such as septic shock and multiorgan dysfunction, which are the main causes of death for IBPA patients. Dyspnea without exertion and diffuse wheezing rales in the lungs are manifestations of severe bronchospasm, which may suggest a local inflammatory status of the airways. The presentation of severe inflammatory status and bronchospasm are not specific for IBPA; however, when they are resistant to appropriate antibiotics and corticosteroids, a diagnosis of IBPA should be suspected.
A local inflammatory reaction can also be observed directly during bronchoscopy, presenting as mucous hyperemia, edema, large amounts of ropy airway secretions, plaque, pseudomembrane formation, and bronchospasm. Further, performing bronchoscopy with microscopic examinations of tracheal or bronchial specimens is the most sensitive diagnostic test for an early diagnosis and treatment of ATB [2]. In our study, four patients had ATB presentations during bronchoscopic analysis, and all of them were confirmed as proven ATB with biopsies of tracheobronchial tissues. A previous study suggested that when IPA is suspected, but culture evaluations of nonbronchoscopic samples alone are negative, intubation and bronchoscopy should be considered [8]. Therefore, bronchoscopy is necessary for establishing an early diagnosis of ATB.

Risks exist for ICU patients in having chest CTs because of their severe conditions and difficulties with transportation. As a result, radiologic data for this population is mainly dependent on bedside CXR. However, unlike immunocompromised patients, early findings on CXR or CT scan for IBPA in CORD patients are nonspecific, and halo signs and cavitations are uncommon $[4,16,20,24]$. Moreover, for some ATB patients, no obvious abnormalities could be found on their chest images. Therefore, it is difficult to establish an early diagnosis of IBPA based on classic manifestations on their chest CTs or CXRs for CORD patients. Further, our study suggested the rapid progression of patching to nodules and consolidations in multiple segments and lobes, which were unresponsive to empiric antibacterial agents. Therefore, the rapid progression on a chest image may be suggestive of suspected IBPA.

In our study, a high proportion of IBPA patients had a positive culture for Aspergillus in the same day (day 1) of ICU admission $(84.6 \%, 11$ of 13). In the 11 cases, no patients had the diagnosis of IA before their admission, no Aspergillus was isolated previous to the ICU admission, and none of these patients received antifungal agents before ICU admission. It means that, probably, these patients were admitted to the unit because of the IA, which can partly explain why the mortality was as high as $69 \%$ in our study.

We realize that this study has limitations. First, to perform multivariate analysis on a small dataset (three predictor variables for 13 IBPA cases) was prone to bias and model overfitting, yielding spurious findings, which made a large $95 \%$ CI for OR in our analysis. Increasing the sample size and collecting more IBPA cases in a further study may avoid this kind of limitation. Second, only four patients had biopsies and were diagnosed as having proven IBPA, which may cause possible misclassification bias. Finally, this is a single-center study, in which a setting may have tremendous overuse of 
antibiotic agents for empiric treatment and prophylaxis. A multicenter prospective study maybe needed in the future to avoid this kind of limitation.

\section{Conclusions}

In conclusion, IBPA is not rare among critically ill CORD patients with ICU admissions, with mortality as high as $69 \%$. IBPA may be suspected in critically ill CORD patients with respiratory failure and clinical and bronchoscopic manifestations of severe infection (high fever, elevated WBC count, low blood pressure and multiorgan dysfunction, sputum ropiness, and plaque formation), bronchospasm (wheeze and dry rales), and rapid progression of radiologic lesions, which are irresponsive to steroids and antibiotics. To avoid misdiagnosis and to establish the microbiologic etiology, early bronchoscopy and tight radiologic follow-up should be performed.

\section{Key messages}

- ATB may be an early stage of IPA and may exist either before or with IPA. Therefore, ATB and IPA might be two phases or manifestations of one entity, invasive bronchopulmonary aspergillosis (IBPA).

- In critically ill CORD patients, before ICU admission, three variables were independent predictors of IBPA: more than three kinds of antibiotics used before the ICU admission, accumulated doses of corticosteroids ( $>350 \mathrm{mg}$ ) received before the ICU admission, and APACHE II scores $>18$.

- In critically ill CORD patients, IBPA may present as respiratory failure and clinical and bronchoscopic manifestations of severe infection, bronchospasm, and rapid progression of radiologic lesions that are irresponsive to steroids and antibiotics.

- IBPA is not rare among critically ill CORD patients with ICU admissions, with mortality as high as $69 \%$.

\section{Additional material}

Additional file 1: Figure S1. Arterial blood gas analysis and blood cell count after RICU admission.

Additional file 2: Figure S2. Biochemical and coagulation test after RICU admission.

Additional file 3: Figure S3. Comparison of the chest radiologic presentation between the two groups.

\footnotetext{
Abbreviations

ABGA: arterial blood gas analysis; APACHE II: the Acute Physiology and Chronic Health Evaluation II; APTT: activated partial thromboplastin time; ATB: Aspergillus tracheobronchitis; BE: base excess; COPD: chronic obstructive pulmonary disease; CORD: chronic obstructive respiratory disease; $C T$ : computed tomography; CXR: chest x-ray; EORTC/MSG: the European Organization for Research and Treatment of Cancer/Mycoses Study Group; GM: galactomannan; IA: invasive aspergillosis; IBPA: invasive bronchial-
}

pulmonary aspergillosis; ICU: intensive care unit; IPA: invasive pulmonary aspergillosis; LRT: lower respiratory tract; WBC: white blood cell.

\section{Acknowledgements}

We acknowledge the efforts of Dr. Lirong Liang for her statistical support. Written consent for publication was obtained from patient case no. 2 of IBPA.

\section{Author details}

${ }^{1}$ Department of Respiratory and Critical Care Medicine, Beijing Institute of Respiratory Medicine, Beijing Chao-Yang Hospital, Capital Medical University, 8 Gongren Tiyuchang South Road, Beijing, 100020, PR China. ${ }^{2}$ Department of Infectious Diseases and Clinical Microbiology, Beijing Institute of Respiratory Medicine, Beijing Chao-Yang Hospital, Capital Medical University, 8 Gongren Tiyuchang South Road, Beijing, 100020, PR China.

\section{Authors' contributions}

All authors made substantial contributions to conception and design, or acquisition of data, or analysis and interpretation of data; reviewed and approved the final manuscript; and contributed significantly to this study. Drs $\mathrm{HH}$ and LD contributed equally to the work. QZ takes full responsibility for the integrity of the submission and publication and was involved in study design. HH had full access to all the data in the study, takes responsibility for the integrity of the data and the accuracy of the data analysis, and was responsible for the data verification, analysis, and drafting of the manuscript. LD had full access to all the data in the study and takes responsibility for the integrity of the data and the accuracy of the data analysis. FL was responsible for the microbiologic examination and the data collection.

\section{Competing interests}

The authors declare that they have no competing interests.

Received: 8 September 2010 Revised: 30 November 2010

Accepted: 6 January 2011 Published: 6 January 2011

\section{References}

1. Pauw BD, Walsh T, Donnelly J, Stevens D, Edwards J, Calandra T, Pappas P, Maertens J, Lortholary O, Kauffman C, Denning D, Patterson T, Maschmeyer G, Bille J, Dismukes W, Herbrecht R, Hope W, Kibbler C, Kullberg B, Marr K, Munoz P, Odds F, Perfect J, Restrepo A, Ruhnke M, Segal B, Sobel J, Sorrell T, Viscoli C, Wingard J, et al: Revised definitions of invasive fungal disease from the European Organization for Research and Treatment of Cancer/Invasive Fungal Infections Cooperative Group and the National Institute of Allergy and Infectious Diseases Mycoses Study Group (EORTC/MSG) Consensus Group. Clin Infect Dis 2008, 46:1813-1821.

2. Tasci S, Glasmacher A, Lentini S, Tschubel K, Ewig S, Molitor E, Sauerbruch T, Luderitz B, Rabe C: Pseudomembranous and obstructive Aspergillus tracheobronchitis: optimal diagnostic strategy and outcome. Mycoses 2006, 49:37-42.

3. Bousquet J, Dahl R, Khaltaev N: Global Alliance against Chronic Respiratory Diseases. Eur Respir J 2007, 29:233-239.

4. Pittet D, Huguenin T, Dharan S, Sztajzel-Boissard J, Ducel G, Thorens J, Auckenthaler R, Chevrolet J: Unusual cause of lethal pulmonary aspergillosis in patients with chronic obstructive pulmonary disease. Am J Respir Crit Care Med 1996, 154:541-544.

5. Crean J, Niederman M, Fein A, Feinsilver S: Rapidly progressive respiratory failure due to Aspergillus pneumonia: a complication of short-term corticosteroid therapy. Crit Care Med 1992, 20:148-150.

6. Rello J, Esandi M, Mariscal D, Gallego M, Domingo C, Valles J: Invasive pulmonary aspergillosis in patients with chronic obstructive pulmonary disease: report of eight cases and review. Clin Infect Dis 1998, 26:1473-1475.

7. Bulpa $P$, Dive A, Sibille $Y$ : Invasive pulmonary aspergillosis in patients with chronic obstructive pulmonary disease. Eur Respir J 2007, 30:782-800.

8. Bulpa P, Dive A, Garrino M, Delos M, Gonzalez M, Evrard P, Glupczynski Y, Installe E: Chronic obstructive pulmonary disease patients with invasive pulmonary aspergillosis: benefits of intensive care? Intensive Care Med 2001, 27:59-67. 
9. Clancy C, Nguyen M: Acute community-acquired pneumonia due to Aspergillus in presumably immunocompetent hosts: clues for recognition of a rare but fatal disease. Chest 1998, 114:629-634.

10. Meersseman W, Vandecasteele SJ, Wilmer A, Verbeken E, Peetermans WE, Wijngaerden EV: Invasive aspergillosis in critically ill patients without malignancy. Am J Respir Crit Care Med 2004, 170:621-625.

11. Xavier MO, Sales Mda P, Camargo Jde J, Pasqualotto AC, Severo LC: Aspergillus niger causing tracheobronchitis and invasive pulmonary aspergillosis in a lung transplant recipient: case report. Rev Soc Bras Med Trop 2008, 41:200-201.

12. Kramer MR, Denning DW, Marshall SE, Ross DJ, Berry G, Lewiston NJ, Stevens DA, Theodore J: Ulcerative tracheobronchitis after lung transplantation: a new form of invasive aspergillosis. Am Rev Respir Dis 1991, 144:552-556.

13. Wu N, Huang Y, Li Q, Bai C, Huang HD, Yao XP: Isolated invasive Aspergillus tracheobronchitis: a clinical study of 19 cases. Clin Microbiol Infect 2010, 16:689-695.

14. Campbell MJ, Clayton YM: Bronchopulmonary aspergillosis: a correlation of the clinical and laboratory findings in 272 patients investigated for bronchopulmonary aspergillosis. Am Rev Respir Dis 1964, 89:186-196.

15. Young RC, Bennett JE, Vogel CL, Carbone PP, DeVita VT: Aspergillosis: the spectrum of the disease in 98 patients. Medicine (Baltimore) 1970, 49:147-173.

16. Garnacho-Montero J, Amaya-Villar R, Ortiz-Leyba C, Leon C, Alvarez-Lerma F, Nolla-Salas J, Iruretagoyena J, Barcenilla F: Isolation of Aspergillus spp. from the respiratory tract in critically ill patients: risk factors, clinical presentation and outcome. Crit Care 2005, 9:R191-R199.

17. Diamond $R$ : Inhibition of monocyte-mediated damage to fungal hyphae by steroid hormones. J Infect Dis 1983, 147:160.

18. Schaffner A: Therapeutic concentrations of glucocorticoids suppress the antimicrobial activity of human macrophages without impairing their responsiveness to gamma interferon. J Clin Invest 1985, 76:1755-1764.

19. Tony TC, Robson GD, Denning DW: Hydrocortisone-enhanced growth of Aspergillus spp.: implications for pathogenesis. Microbiology 1994, 140:2475-2479.

20. Muquim A, Dial S, Menzies D: Invasive aspergillosis in patients with chronic obstructive pulmonary diseases. Can Respir J 2005, 12:199-204.

21. Guinea J, Torres-Narbona M, Gijon P, Munoz P, Pozo F, Pelaez T, de Miguel J, Bouza E: Pulmonary aspergillosis in patients with chronic obstructive pulmonary disease: incidence, risk factors, and outcome. Clin Microbiol Infect 2010, 16:870-877.

22. Kaiser $L$, Huguenin $T$, Lew $P$, Chapuis B, Pittet $D$ : Invasive aspergillosis: clinical features of 35 proven cases at a single institution. Medicine (Baltimore) 1998, 77:188-192.

23. Samarakoon $P$, Soubani $A$ : Invasive pulmonary aspergillosis in patients with COPD: a report of five cases and systematic review of the literature. Chron Respir Dis 2008, 5:19-21.

24. Zhan Q, He H, Tong Z, Li F, Sun B, Wang C: Clinical features of invasive pulmonary aspergillosis in critically ill patients with chronic respiratory diseases. Zhonghua Jie He He Hu Xi Za Zhi 2008, 31:282-286.

doi:10.1186/cc9402

Cite this article as: He et al:: Clinical features of invasive bronchialpulmonary aspergillosis in critically ill patients with chronic obstructive respiratory diseases: a prospective study. Critical Care 2011 15:R5.

\section{Submit your next manuscript to BioMed Central and take full advantage of:}

- Convenient online submission

- Thorough peer review

- No space constraints or color figure charges

- Immediate publication on acceptance

- Inclusion in PubMed, CAS, Scopus and Google Scholar

- Research which is freely available for redistribution

Submit your manuscript at www.biomedcentral.com/submit
Biomed Central 